\title{
Universal random matrix correlations of ratios of characteristic polynomials at the spectral edges
}

\author{
G. AKEMAnN ${ }^{1}$ and Y.V. Fyodorov ${ }^{2}$ \\ ${ }^{1}$ Service de Physique Théorique, CEA/DSM/SPhT Saclay \\ Unité de recherche associée au CNRS \\ F-91191 Gif-sur-Yvette Cedex, France \\ ${ }^{2}$ Department of Mathematical Sciences, Brunel University \\ Uxbridge, UB8 3PH, United Kingdom
}

\begin{abstract}
It has been shown recently [10 that Cauchy transforms of orthogonal polynomials appear naturally in general correlation functions containing ratios of characteristic polynomials of random $N \times N$ Hermitian matrices. Our main goal is to investigate the issue of universality of large $\mathrm{N}$ asymptotics for those Cauchy transforms for a wide class of weight functions. Our analysis covers three different scaling regimes: the "hard edge", the "bulk" and the "soft edge" of the spectrum, thus extending the earlier results known for the bulk. The principal tool is to show that for finite matrix size $N$ the auxiliary "wave functions" associated with the Cauchy transforms obey the same second order differential equation as those associated with the orthogonal polynomials themselves.
\end{abstract}

SPhT T03/036 


\section{Introduction}

Characteristic polynomials are important objects in Random Matrix Theory (RMT) and enjoy a wide range of applications in various fields of physics and mathematics. To name only a few, we mention the applications to Quantum Chromodynamics (QCD) [1], Quantum Chaos 2] as well as their role in elucidating properties of the Riemann zeta function along the critical line [3, 4. In fact, products of ratios of the characteristic polynomials at different values of the spectral parameter provide the most general generating functional for all spectral correlation functions in RMT. Characteristic polynomials are also useful in providing a direct link between the RMT description and the effective field theory such as Chiral Perturbation Theory $(\chi \mathrm{PT})$ in the application to QCD [ ㅎ, 6, 7]. There, products of the ratios of characteristic polynomials enjoy a direct interpretation as partitions functions including fermionic and bosonic degrees of freedom. However, these objects are very difficult to obtain in full generality from the finite volume limit of $\chi \mathrm{PT}$, where one has to parameterize and calculate supergroup integrals [6]. Alternatively, one has to perform replica calculations exactly [7, a possibility opened due to recent progress in understanding the analytical structure of the moments [8]. From this side, correlation functions of characteristic polynomials recently calculated within RMT [9, 10] provide the result yet to be obtained [1] directly from the field-theoretic calculations.

An important issue for all RMT calculations is the question of universality. By this one usually understands that the predictions of RMT for a given object are insensitive to the details of the particular models. When this property holds, it must be a consequence of the common underlying symmetry of that class of the models. This type of argument validates applying RMT results to systems of varying nature in all the diverse situations mentioned above.

The character of universality depends essentially on the scale in the spectrum one is interested in, as well as on the nature of the singularity at the support of the spectrum (the so-called "edges" of the spectrum). In the following we will be only concerned with the scales comparable with the mean level spacing. This issue is widely known as that of "microscopic" universality. Suppose first one is interested in such quantities as the correlation function of eigenvalue densities, or the distribution of the largest/smallest eigenvalue, or some related objects. Then restricting oneself to the class of random matrix measures invariant with respect to the change of basis (here the unitary invariant ensembles) the quantity of interest can be expressed as a determinant containing kernels made of polynomials orthogonal with respect to that measure [12. In this way studying universality amounts mainly to investigating asymptotics of the orthogonal polynomials in various regimes, and is well established by now both on physical [13, 14, 15, 16, 17, 18, 19] as well as mathematical [20, 21, 22, 23] level of rigor.

On the other hand, more general correlation functions containing products and ratios of the characteristic polynomials (in particular, those used to generate all the eigenvalue correlations by differentiation and taking the discontinuity of the resolvents) attracted considerable attention only recently [7, 9, 10, 11, 25]. While for the products only the universality follows quite straightforwardly from the mentioned results [13, 14, 15, 16, 20, 21, 22, 23, (see also 24]), the universality of expressions containing inverse powers or ratios was properly understood only in the bulk of the spectrum [25].

There are several possibilities to calculate correlation functions containing ratios of characteristic polynomials. For the case of the Gaussian measure the only tool until quite recently was the exploitation of anticommuting Grassmann variables (the method of "supersymmetry" due to Efetov [26], or its variants [27, 28, 2, 9, 10]). An alternative for general unitary-invariant measures was discovered quite recently by Fyodorov and Strahov [10, 25] and resorts again to the orthogonal polynomial method. An important new feature, however, is that as soon as inverse powers of characteristic polynomials are taken into consideration, one has to deal not only with the orthogonal polynomials but also with their so-called Cauchy transforms. More precisely, a very general result - valid for all invariant ensembles of finite-size complex Hermitian matrices [10] - reduces arbitrary correlation function containing ratios of 
characteristic polynomials to a determinant made of orthogonal polynomials and their Cauchy transforms. The universality of the corresponding expression in the microscopic limit in the "bulk" of the spectrum was rigorously demonstrated by Strahov and Fyodorov [25] by using the Riemann-Hilbert approach. Those authors however considered neither the "soft" nor the "hard" edge of the spectrum, the latter being a characteristic feature of the so-called "chiral" version of the unitary ensemble 29, 1].

Our goal in the present paper is to employ an alternative method and to obtain universal asymptotics for all three regimes, including spectral edges of both types. This is particularly important in view of the application to QCD and the role of characteristic polynomials played there (see. e.g. in [7. 11] and references therein). The method we are going to exploit to extract universal features of the correlation functions is based on the works by Kanzieper and Freilikher [30. The clear advantage of this technique is its explicit form and conceptual simplicity, although it lacks the rigor and vigor of the Riemann-Hilbert technique (and in fact makes use of some facts proved by the latter). First we will show the existence of an exact second order differential equation satisfied by the Cauchy transform of the orthogonal polynomials for a large class of measures. Then by considering the microscopic large- $N$ limit of that differential equation we can demonstrate their universality at the origin ("hard edge"), in the bulk and at the soft edges of the spectrum. The procedure follows exactly the same lines as for the orthogonal polynomials themselves (see [30]), since in both cases we have to deal with the very same equation. The universality of asymptotics for the general expressions [10] of arbitrary ratios of characteristic polynomials then follow. It is necessary to mention that the method implicitly uses an assumption of the existence of a single-valued function arising in a certain limit for recursion coefficients relating orthogonal polynomials of different order. This was indeed shown to be true in the case of a spectrum supported on a single interval in the framework of the Riemann-Hilbert technique.

The article is organized as follows. In sect. 2 we give the main definitions and recall the main result of [10]. In the following sect. B] we derive a differential equation for the wave functions associated with the Cauchy transforms for finite $N$ and show that it is identical to that for the wave functions of orthogonal polynomials. For convenience we treat the unitary ensemble (UE) first and translate to the chiral unitary ensemble (chUE) later on. Sect. 4 deals with the large- $N$ limit yielding universal results for both unitary and chiral unitary ensemble in all three microscopic regimes. We close with the discussion and open questions in sect. [5.

\section{Definitions}

In this section we introduce the main objects we wish to study in the present paper. The matrix model partition function with respect to which we calculate expectation values is given by the following integral over the (unitary invariant) Haar measure for matrices $H$ :

$$
\mathcal{Z}_{N} \sim \int_{D}[d H]_{N \times N} w(H) \sim \int_{D} \prod_{k=1}^{N} d \lambda_{k} w\left(\lambda_{k}\right) \Delta_{N}(\Lambda)^{2}
$$

The matrix $H$ can be either complex Hermitian, representing a general unitary invariant ensemble without chiral symmetry, or it can be decomposed into a product of complex rectangular matrices $W$ of size $N \times(N+\nu), H=W^{\dagger} W$, representing the chUE. In the first case the matrices $H$ can be diagonalized by a unitary transformation with real eigenvalues $\Lambda=\operatorname{diag}\left(\lambda_{1}, \ldots, \lambda_{N}\right)$ are distributed according to a weight function $w(\lambda)$ with support on $\mathbb{R}$. In the second case we deal rather with real positive singular values of the matrices $W$ with the support being $\mathbb{R}_{+}$, respectively. We have also employed the notation $\Delta_{N}(\Lambda) \equiv \prod_{i>j}^{N}\left(\lambda_{i}-\lambda_{j}\right)$ for the Vandermonde determinant. While the results cited in this section hold for general weight functions with convergent moments on some domain 
$D \subseteq \mathbb{R}$, we will concentrate mainly on weights of the form $w(\lambda)=\exp [-N V(\lambda)]$ in the following sections. The potential $V(x)$ may contain both an (even) polynomial part and a logarithmic part.

We will be interested in extracting the large- $N$ universal behavior of the correlation functions defined via expectation values of arbitrary ratios of characteristic polynomials:

$$
\mathcal{K}_{N}\left(M_{f}, M_{b}\right) \equiv\left\langle\frac{\prod_{j=1}^{L} \operatorname{det}\left(m_{j}^{f} \mathbf{1}_{N}+H\right)}{\prod_{j=1}^{M} \operatorname{det}\left(m_{j}^{b} \mathbf{1}_{N}+H\right)}\right\rangle .
$$

In the context of QCD the matrices $M_{f}=\operatorname{diag}\left(m_{1}^{f}, \ldots, m_{L}^{f}\right), M_{b}=\operatorname{diag}\left(m_{1}^{b}, \ldots, m_{M}^{b}\right)$ are said to contain the fermionic $(f)$ and bosonic $(b)$ mass terms. The expectation value of ratios of characteristic polynomials is known as the supersymmetric partition function. It may be used to calculate correlation functions of the eigenvalue densities and serves as a general generating function for multi-point resolvent operators.

In order to give rise to positive definite partition functions in the case of the UE the masses have to be chosen purely imaginary and occur in pairs of complex conjugates. In this way only positive definite terms appear, $\operatorname{det}(i m+H) \operatorname{det}(-i m+H)=\prod_{k}^{N}\left(m^{2}+\lambda_{k}^{2}\right)$. In the chUE each determinant factor is positive provided we parameterize $\operatorname{det}\left(m^{2}+W^{\dagger} W\right)=\prod_{k}^{N}\left(m^{2}+\lambda_{k}\right), \lambda_{k} \geq 0$. At the same time, from the mathematical point of view there is no need to impose any restrictions on masses in eq. (2.2), apart from requiring infinitesimal imaginary parts for all $m_{j}^{b}$.

As it has been shown in 10] for general weight functions and finite size $N$ the generating function eq. (2.2) can be written as a determinant of size $M+L$ containing polynomials orthogonal with respect to the weight $w(\lambda)$. Let us define the monic orthogonal polynomials $\pi_{n}(\lambda)=\lambda^{n}+\mathcal{O}\left(\lambda^{n-1}\right)$

$$
\int_{D} d \lambda w(\lambda) \pi_{n}(\lambda) \pi_{m}(\lambda)=\delta_{n m} f_{n}
$$

with normalization factors $f_{n}$ uniquely defined through the Gram-Schmidt orthogonalization. ${ }^{1}$ The second building block are the Cauchy transforms of those polynomials which appear only as long as $M>0$

$$
\vartheta_{n}(\epsilon) \equiv \frac{1}{2 \pi i} \int_{D} d \lambda \frac{w(\lambda)}{\lambda-\epsilon} \pi_{n}(\lambda), \quad \Im m(\epsilon) \neq 0 .
$$

The result derived in [10] then reads ${ }^{2}$

$$
\mathcal{K}\left(M_{f}, M_{b}\right) \propto \frac{1}{\Delta\left(M_{b}\right) \triangle\left(\hat{M}_{f}\right)} \operatorname{det}\left|\begin{array}{cccc}
\vartheta_{N-M}\left(-m_{1}^{b}\right) & \vartheta_{N-M+1}\left(-m_{1}^{b}\right) & \ldots & \vartheta_{N+L-1}\left(-m_{1}^{b}\right) \\
\vdots & \vdots & & \vdots \\
\vartheta_{N-M}\left(-m_{M}^{b}\right) & \vartheta_{N-M+1}\left(-m_{M}^{b}\right) & \ldots & \vartheta_{N+L-1}\left(-m_{M}^{b}\right) \\
\pi_{N-M}\left(-m_{1}^{f}\right) & \pi_{N-M+1}\left(-m_{1}^{f}\right) & \ldots & \pi_{N+L-1}\left(-m_{1}^{f}\right) \\
\vdots & \vdots & & \vdots \\
\pi_{N-M}\left(-m_{L}^{f}\right) & \pi_{N-M+1}\left(-m_{L}^{f}\right) & \ldots & \pi_{N+L-1}\left(-m_{L}^{f}\right)
\end{array}\right| .
$$

\footnotetext{
${ }^{1}$ For weight functions on $D=\mathbb{R}$ the corresponding norms can be calculated recursively solving $n f_{n-1}=$ $-\int_{-\infty}^{\infty} d \lambda w^{\prime}(\lambda) \pi_{n}(\lambda) \pi_{n-1}(\lambda)$ for even potentials plus an additional equation for potentials without parity.

${ }^{2}$ The object eq. 2.5 can be given a meaning for real values of the mass parameters $m_{l}^{b}$ as well, by choosing the principle value in eq. (2.4). However, to use it as a generating functional we are precisely interested in its discontinuities in the complex plane.
} 


\section{A differential equation for the orthogonal polynomials and their Cauchy transforms}

As is well known [13, 30, 22] it is convenient to relate to the system of orthogonal polynomials $\pi_{n}(\lambda)$ a system of associated objects:

$$
\psi_{n}(\lambda) \equiv \exp \left[-\frac{N}{2} V_{\alpha}(\lambda)\right] \frac{\pi_{n}(\lambda)}{\sqrt{f_{n}}} .
$$

frequently called the "wave functions". The name has its origin in two properties: (i) the functions $\psi_{n}(\lambda)$ obey a closed second order differential equation for finite $N$ which can be interpreted as a kind of one-dimensional Schroedinger equation and (ii) the functions are orthonormal. The first property can be shown, in particular, by the formalism introduced by Kanzieper and Freilikher [30, to which we refer an interested reader for a detailed review. Below we exploit the same formalism to infer properties of the Cauchy transforms of the orthonormal polynomials, after suitable redefinition of the wavefunctions. This will enable us to treat the Cauchy transforms on equal footing with the polynomials themselves when extracting the universal properties in the microscopic large- $N$ limit. The wavefunctions of the orthogonal polynomials and of their Cauchy transforms will be then two independent solutions of the second order differential equation, with the latter being singular at the origin.

Due to an intimate relation between the polynomials of the UE and the chUE for general weights $w(\lambda)$ we can restrict ourselves to treat only the UE in greater detail in the following subsection. Once our results are established those for the chUE will follow rather straightforwardly, as discussed in in the subsection 3.2

\subsection{The Unitary Ensemble}

For the UE the eigenvalues of the complex Hermitian matrix $H$ are distributed along the full real line. To be specific let us introduce the following parameterization of the weight function

$$
w(\lambda) \equiv \lambda^{2 \alpha} \exp [-N V(\lambda)] \equiv \exp \left[-N V_{\alpha}(\lambda)\right]
$$

Here we have introduced the potential

$$
V_{\alpha}(\lambda) \equiv V(\lambda)-\frac{2 \alpha}{N} \ln (\lambda), \quad V(\lambda)=\sum_{k=1}^{d / 2} \frac{g_{2 k}}{2 k} \lambda^{2 k}
$$

with an even polynomial part $V(\lambda)$ of arbitrary but fixed even degree $d$. In order to apply the formalism of [30] we switch from monic polynomials $\pi_{n}(x)$ to orthonormal polynomials,

$$
\delta_{k l}=\int_{-\infty}^{\infty} d \lambda w(\lambda) p_{k}(\lambda) p_{l}(\lambda), \quad p_{k}(\lambda)=\pi_{k}(\lambda) / \sqrt{f_{k}} .
$$

The potential in eq. (3.3) being even, the polynomials possess the following parity property:

$$
p_{n}(-\lambda)=(-1)^{n} p_{n}(\lambda) .
$$

As is well known (see e.g. 22, 30] and references therein), all orthogonal polynomials on the real line or a subset of it satisfy a three-step recursion relation

$$
\lambda p_{n}(\lambda)=c_{n+1} p_{n+1}(\lambda)+c_{n} p_{n-1}(\lambda),
$$


where the middle term vanishes because of the parity. The recursion coefficients can be obtained from the normalization factors $f_{n}$ of the polynomials as:

$$
c_{n}=\sqrt{\frac{f_{n}}{f_{n-1}}} .
$$

The second operation, which together with the multiplication by $\lambda$ in eq. (3.6) closes on the ring of orthogonal polynomials, is differentiation over $\lambda$. Following [30] it can be most conveniently written as

$$
p_{n}^{\prime}(\lambda) \equiv A_{n}(\lambda) p_{n-1}(\lambda)-B_{n}(\lambda) p_{n}(\lambda)
$$

Here, the functions $A_{n}(\lambda)$ and $B_{n}(\lambda)$ are defined as

$$
\begin{aligned}
A_{n}(\lambda) & \equiv N c_{n} \int_{-\infty}^{\infty} d \eta \frac{w(\eta)}{\eta-\lambda}\left[V_{\alpha}^{\prime}(\eta)-V_{\alpha}^{\prime}(\lambda)\right] p_{n}(\eta)^{2} \\
& =N c_{n} \int_{-\infty}^{\infty} d \eta \frac{w(\eta)}{\eta-\lambda}\left[V^{\prime}(\eta)-V^{\prime}(\lambda)\right] p_{n}(\eta)^{2} \\
B_{n}(\lambda) & \equiv N c_{n} \int_{-\infty}^{\infty} d \eta \frac{w(\eta)}{\eta-\lambda}\left[V_{\alpha}^{\prime}(\eta)-V_{\alpha}^{\prime}(\lambda)\right] p_{n}(\eta) p_{n-1}(\eta) \\
& \equiv B_{n}^{\mathrm{reg}}(\lambda)+\left(1-(-1)^{n}\right) \frac{\alpha}{\lambda} .
\end{aligned}
$$

Because of parity the singular $\alpha$-dependent term drops out in $A_{n}(\lambda)$. Inserting the explicit form of the potential from eq. (3.3) it can be easily seen that $A_{n}(\lambda)$ and the regular part $B_{n}^{\text {reg }}(\lambda)$ of $B_{n}(\lambda)$ are polynomials of degree $d-2$ and $d-3$ respectively. From their definition one can easily show the following identity to be valid [15]:

$$
B_{n}(\lambda)+B_{n-1}(\lambda)+N V_{\alpha}^{\prime}(\lambda)=\frac{\lambda}{c_{n-1}} A_{n-1}(\lambda)
$$

This identity, when combined with the two properties of the polynomials - the recursion relation eq. (3.6) and the differentiation eq. (3.8) - suffices to show that the following second order differential equation is satisfied by the wavefunctions defined in Eq.(3.1):

$$
\psi_{n}^{\prime \prime}(\lambda)-F_{n}(\lambda) \psi_{n}^{\prime}(\lambda)+G_{n}(\lambda) \psi_{n}(\lambda)=0
$$

where

$$
\begin{aligned}
F_{n}(\lambda) \equiv & \frac{A_{n}^{\prime}(\lambda)}{A_{n}(\lambda)} \\
G_{n}(\lambda) \equiv & \frac{c_{n}}{c_{n-1}} A_{n}(\lambda) A_{n-1}(\lambda)-\left(B_{n}(\lambda)+\frac{N}{2} V_{\alpha}^{\prime}(\lambda)\right)^{2} \\
& +\left(B_{n}(\lambda)+\frac{N}{2} V_{\alpha}^{\prime}(\lambda)\right)^{\prime}-\frac{A_{n}^{\prime}(\lambda)}{A_{n}(\lambda)}\left(B_{n}(\lambda)+\frac{N}{2} V_{\alpha}^{\prime}(\lambda)\right) .
\end{aligned}
$$

Here ' denotes the derivative with respect to $\lambda$ everywhere. The differential equation Eq.(3.12) holds for finite- $N$ and arbitrary weight $w(\lambda)$ corresponding to a given polynomial $V(\lambda)$ of degree $d$ and parameter $\alpha>-1 / 2$. The fact that $\alpha$ can be real will enable us to translate most easily the results for the UE obtained below to the chUE in the next subsection. 
Our goal is to derive a similar differential equation for the Cauchy transform at finite- $N$. In terms of the orthonormal polynomials they are given by

$$
h_{n}(\epsilon) \equiv \frac{1}{2 \pi i} \int_{-\infty}^{+\infty} d \lambda \frac{w(\lambda)}{\lambda-\epsilon} p_{n}(\lambda), \quad \Im \mathrm{m}(\epsilon) \neq 0
$$

This defines in fact two functions, one in the upper and one in the lower half plane for positive or negative imaginary part, respectively. Each of it can be extended to the other half plane using the relation

$$
h_{n}(-\epsilon)=(-1)^{n+1} h_{n}(\epsilon) .
$$

which follows easily from the parity of the polynomials, eq. 3.5. We note that the Cauchy transform has the opposite parity than the corresponding polynomial. Let us stress that the transformation (3.16) does not relate the two different functions defined in eq. (3.15).

In the following we show that the object

$$
\tilde{h}_{n}(\epsilon) \equiv \exp \left[+N V_{\alpha}(\epsilon)\right] h_{n}(\epsilon)
$$

satisfies the same recursion relation eq. (3.6) and differentiation relation eq. (3.8) as the corresponding orthogonal polynomial $p_{n}(\lambda)$, provided that $n>d-1$. As an immediate consequence the wavefunction for the Cauchy transform defined as

$$
\chi_{n}(\epsilon) \equiv \exp \left[-\frac{N}{2} V_{\alpha}(\epsilon)\right] \tilde{h}_{n}(\epsilon)=\exp \left[+\frac{N}{2} V_{\alpha}(\epsilon)\right] h_{n}(\epsilon)
$$

satisfies the second order differential equation identical to Eq. (3.12) at finite- $n$, provided the condition $n>d-2$. The corresponding solution of the differential equation at finite and large- $n$ will then have to be chosen according to the appropriate boundary condition for $\psi_{n}$ and $\chi_{n}$, respectively.

Let us first consider multiplication on $h_{n}(\epsilon)$ for $n>0$ :

$$
\begin{aligned}
\epsilon h_{n}(\epsilon) & =\frac{1}{2 \pi i} \int_{-\infty}^{+\infty} d \lambda \frac{w(\lambda)}{\lambda-\epsilon} \epsilon p_{n}(\lambda) \\
& =\frac{-1}{2 \pi i} \int_{-\infty}^{+\infty} d \lambda w(\lambda) p_{n}(\lambda)+\frac{1}{2 \pi i} \int_{-\infty}^{+\infty} d \lambda \frac{w(\lambda)}{\lambda-\epsilon}\left[c_{n+1} p_{n+1}(\lambda)+c_{n} p_{n-1}(\lambda)\right] \\
& =c_{n+1} h_{n+1}(\epsilon)+c_{n} h_{n-1}(\epsilon), n>0 .
\end{aligned}
$$

At the first step we have added and subtracted $\lambda$ to cancel the denominator and used the recursion relation eq. (3.6) $)$. At the second step we used that $p_{n}(\lambda)$ is orthogonal to $p_{0}(\lambda)=$ const for $n>0$. The fact that the recursion Eq. (3.19) also holds for $\tilde{h}_{n}(\epsilon)$ as well as for $\chi_{n}(\epsilon)$ is an immediate consequence.

The next step is to consider differentiation acting on $\tilde{h}_{n}(\epsilon)$ for $n>d-2$ :

$$
\begin{aligned}
\partial_{\epsilon} \tilde{h}_{n}(\epsilon)= & \frac{1}{2 \pi i} \int_{-\infty}^{+\infty} d \lambda \frac{\mathrm{e}^{N\left(V_{\alpha}(\epsilon)-V_{\alpha}(\lambda)\right)}}{\lambda-\epsilon}\left[N\left(V_{\alpha}^{\prime}(\epsilon)-V_{\alpha}^{\prime}(\lambda)\right) p_{n}(\lambda)+p_{n}^{\prime}(\lambda)\right] \\
= & \frac{1}{2 \pi i} \int_{-\infty}^{+\infty} d \lambda \mathrm{e}^{N\left(V_{\alpha}(\epsilon)-V_{\alpha}(\lambda)\right)}\left[N \frac{V^{\prime}(\epsilon)-V^{\prime}(\lambda)}{\lambda-\epsilon} p_{n}(\lambda)-\frac{2 \alpha}{\epsilon \lambda} p_{n}(\lambda)\right. \\
& \left.+\frac{A_{n}(\lambda)}{\lambda-\epsilon} p_{n-1}(\lambda)-\frac{B_{n}^{\mathrm{reg}}(\lambda)+\left(1-(-1)^{n}\right) \alpha \lambda^{-1}}{\lambda-\epsilon} p_{n}(\lambda)\right] \\
= & \frac{1}{2 \pi i} \int_{-\infty}^{+\infty} d \lambda \mathrm{e}^{N\left(V_{\alpha}(\epsilon)-V_{\alpha}(\lambda)\right)}\left[-\frac{2 \alpha}{\epsilon \lambda} p_{n}(\lambda)+\frac{A_{n}(\epsilon)}{\lambda-\epsilon} p_{n-1}(\lambda)\right. \\
& \left.-\frac{B_{n}^{\mathrm{reg}}(\epsilon)+\left(1-(-1)^{n}\right) \alpha \epsilon^{-1}}{\lambda-\epsilon} p_{n}(\lambda)+\frac{\alpha\left(1-(-1)^{n}\right)}{\epsilon \lambda} p_{n}(\lambda)\right] \\
= & A_{n}(\epsilon) \tilde{h}_{n-1}(\epsilon)-\left[B_{n}^{\mathrm{reg}}(\epsilon)+\left(1-(-1)^{n}\right) \frac{\alpha}{\epsilon}\right] \tilde{h}_{n}(\epsilon), \quad n>d-2 .
\end{aligned}
$$


Here we first have used the relation $\partial_{\epsilon} \frac{1}{\epsilon-\lambda}=-\partial_{\lambda} \frac{1}{\epsilon-\lambda}$ and performed the integration by parts. The second step spells out the definition of $V_{\alpha}(\lambda)$ and exploits Eq. (3.8) for $p_{n}^{\prime}(\lambda)$. We can now use that $\left(V^{\prime}(\epsilon)-V^{\prime}(\lambda)\right) /(\lambda-\epsilon)$ is a polynomial of order $d-2$ and thus orthogonal to $p_{n}(\lambda)$ for $n>d-2$. The same argument helps to rewrite $A_{n}(\lambda) /(\lambda-\epsilon)=\left(A_{n}(\lambda)-A_{n}(\epsilon)\right) /(\lambda-\epsilon)+A_{n}(\epsilon) /(\lambda-\epsilon)$ and to drop the first term, which is again a polynomial (this times of the order $d-3$ ) and thus orthogonal to $p_{n}(\lambda)$. We can repeat the same steps for $B_{n}^{\text {reg }}(\lambda)$ and the poles $1 / \lambda$. The remaining terms $\sim 1 /(\lambda \epsilon)$ require a little bit more careful treatment. For $n$ even $p_{n}(\lambda) / \lambda$ is odd and thus the corresponding integral vanishes identically. For $n$ odd the last term $\sim\left(1-(-1)^{n}\right)$ gives a contribution that precisely cancels the first one. We thus finally arrive at the same relation for differentiation of $\tilde{h}_{n}(\epsilon)$ as that given by eq. (3.8). Consequently, Eqs. (3.19), (3.20) and (3.18) imply

$$
\chi_{n}^{\prime \prime}(\lambda)-F_{n}(\lambda) \chi_{n}^{\prime}(\lambda)+G_{n}(\lambda) \chi_{n}(\lambda)=0 .
$$

Thus, the wave functions associated with the orthogonal polynomials and their Cauchy transforms satisfy the same differential equation, provided that condition $n>d-2$ holds. This constraint is not an obstacle for large but finite- $n$, for any given potential $V(\lambda)$ with a fixed finite degree $d$.

The virtue of our analysis at finite $n$ is that it obviously translates all the universality proofs carried out at the origin, in the bulk and at the edge of the spectrum for the orthogonal polynomials to their Cauchy transforms. We will concentrate on the the large- $n$ limit in the next section 4 , but first we repeat the above analysis for the chiral case of chUE.

\subsection{The chiral Unitary Ensemble}

The eigenvalues for chUE are distributed on the positive real line $\mathbb{R}_{+}$and governed by the following chiral weight function:

$$
w^{c h}(\lambda) \equiv \lambda^{\alpha} \exp \left[-N V^{c h}(\lambda)\right] \equiv \exp \left[-N V_{\alpha}^{c h}(\lambda)\right]
$$

Here the potential $V_{\alpha}^{c h}(\lambda)$ is of degree $d / 2$, with $d$ even, and is defined as

$$
V_{\alpha}^{c h}(\lambda) \equiv \sum_{k=1}^{d / 2} \frac{g_{2 k}}{k} \lambda^{k}-\frac{\alpha}{N} \ln (\lambda) .
$$

The orthogonality relation for the corresponding orthogonal polynomials denoted as $p_{k, c h}^{(\alpha)}(\lambda) \operatorname{reads}$

$$
\delta_{k l}=\int_{0}^{\infty} d \lambda w^{c h}(\lambda) p_{k, c h}^{(\alpha)}(\lambda) p_{l, c h}^{(\alpha)}(\lambda)
$$

Here we explicitly display the dependence on the parameter $\alpha$. Substituting $\lambda^{2}=y$ one can easily see that the orthogonal polynomials of the chUE can be obtained from those of the UE by simple replacement of the argument [18:

$$
p_{n, c h}^{(\alpha)}\left(\lambda^{2}\right)=p_{2 n}^{(\alpha+1 / 2)}(\lambda) .
$$

Here we have to appropriately identify the corresponding potential as

$$
V^{c h}\left(y^{2}\right)=2 V(y)
$$

which effectively means that the non-chiral polynomials are taken at values $2 N$. In fact, one can look at the relation Eq. (3.25) as a generalization of the well-known relation between generalized Laguerre 
and Hermite polynomials. Consequently, there is no need to repeat the whole analysis of the previous subsection but one can simple read off the chiral orthogonal polynomials and their asymptotics from the same differential equation (3.12) for even $N$ at shifted value of $\alpha$.

Let us now show that the Cauchy transforms of the chiral orthogonal polynomials relate to those of the non-chiral ones as well. We first define the Cauchy transform for the chiral case as

$$
h_{n, c h}^{(\alpha)}(\epsilon) \equiv \frac{1}{2 \pi i} \int_{0}^{\infty} d \lambda \frac{w^{c h}(\lambda)}{\lambda-\epsilon} p_{n, c h}^{(\alpha)}(\lambda), \quad \Im m(\epsilon) \neq 0 .
$$

Substituting $\lambda^{2}=y$ and inserting the relation (3.25) we obtain

$$
\begin{aligned}
h_{n, c h}^{(\alpha)}(\epsilon) & =\frac{1}{2 \pi i} \int_{-\infty}^{+\infty} d y \frac{y}{y^{2}-\epsilon} \mathrm{e}^{-N V_{\alpha}^{c h}\left(y^{2}\right)} p_{n, c h}^{(\alpha)}\left(y^{2}\right) \\
& =\frac{1}{2 \pi i} \int_{-\infty}^{+\infty} d y \frac{1}{y^{2}-\epsilon} \mathrm{e}^{-N V_{\alpha+1 / 2}^{c h}\left(y^{2}\right)} p_{2 n}^{(\alpha+1 / 2)}(y),
\end{aligned}
$$

where we have used the parity of the non-chiral polynomial $p_{2 n}^{(\alpha+1 / 2)}(y)$. On the other hand we can rewrite the Cauchy transform of the non-chiral ensemble eq. (2.4) with an even index $2 n$ as

$$
h_{2 n}^{(\alpha)}(\epsilon)=\frac{1}{2 \pi i} \int_{-\infty}^{+\infty} d y \frac{\epsilon}{y^{2}-\epsilon^{2}} \mathrm{e}^{-2 N V_{\alpha}(y)} p_{2 n}^{(\alpha)}(y) .
$$

Comparing eqs. (3.29) and (3.30) together with eq. (3.26) we thus arrive at

$$
h_{n, c h}^{(\alpha)}\left(\epsilon^{2}\right)=\frac{1}{\epsilon} h_{2 n}^{(\alpha+1 / 2)}(\epsilon),
$$

where the right hand side has to be taken at $2 N$ as for the polynomials themselves. Consequently the same analysis for the "chiral" Cauchy transforms follows from the same differential equation (3.21).

We note that while the Cauchy transform eq. (3.27) defines a single function with a cut along the positive real line $\mathbb{R}_{+}$, the second transform in eq. (3.28) defines again two functions in the upper and lower half plane as in eq. (2.4). It is in this latter picture on $\mathbb{R}$ that the results of the UE can be translated to the chUE. This is why in the following section we will always give two different solutions for the chiral Cauchy transforms as well.

\section{Universality}

In this section we demonstrate the universality of the asymptotic polynomials and their Cauchy transform in the microscopic limit, which then translates to all correlation functions of characteristic polynomials through eq. (2.5). The limit is called microscopic since it includes a certain rescaling of the eigenvalues with an appropriate power of the parameter $N$, according to the spectral region we investigate. We will first study the microscopic limit at the origin (frequently called the "hard edge"). This case is singled out due to the presence of logarithms in the potential. The correlations in the bulk of the spectrum then follow easily. Furthermore we also study the edge of the spectrum defined by the endpoints of the support of the macroscopic or smoothed spectral density at large $N$ ("soft edge"). Here we will restrict ourselves to the cases where the spectrum support is a simply connected interval, the so-called one-cut phase. We will also stay away from multicritical points, where the macroscopic density develops extra zeros at the boundary of spectra or inside the support. This amounts to requiring the limiting functions for $A_{N}(\lambda)$ to be nonvanishing on the support as well (see eq. (4.3) below). While such multicritical cases can be, in principle, analyzed in the framework 
of the same approach [18, 17, 31] the issue becomes much more subtle due to subleading terms in the large- $N$ expansion.

Our first step is to derive the large- $N$ limit of the differential equations (3.12) and (3.21). Our assumption of the one-cut support is equivalent to the fact that in the large- $N$ limit the recursion coefficients $c_{n}$ in eq. (3.7) approach a single valued function

$$
\lim _{n \rightarrow \infty} c_{n}=c(t=n / N) .
$$

While in the earlier physical matrix model literature this assumption was introduced as an ansatz supported by numerical evidence, it has been proved rigorously in the recent mathematical literature 21]. Eq. (4.1) implies the following asymptotic form for the functions $A_{N}(\lambda)$ and $B_{N}(\lambda)$ in the differential equation [30]:

$$
\begin{aligned}
\lim _{N \rightarrow \infty} \frac{1}{N} A_{N}(\lambda) & \equiv A(\lambda)=\frac{a}{2 \pi} \int_{-a}^{a} d t \frac{t V^{\prime}(t)-\lambda V^{\prime}(\lambda)}{t^{2}-\lambda^{2}} \frac{1}{\sqrt{a^{2}-t^{2}}} \\
& =\pi \rho(\lambda) \frac{a}{\sqrt{a^{2}-\lambda^{2}}}, \quad \lambda \in(-a, a) .
\end{aligned}
$$

Here $a=\lim _{N \rightarrow \infty} 2 c_{N}$ is the endpoint of the spectral support. For convenience we have also presented the relation to the macroscopic density of eigenvalues denoted by $\rho(\lambda)$. Using the recursion relation eq. (3.6) it can be easily seen that $A(\lambda)$ remains a polynomial of degree $d-2$, but the coefficients get smoothened by taking the large- $N$ limit. This corresponds to the one cut phase through eq. (4.3), with the nonpolynomial part of $\rho(\lambda) \sim \sqrt{a^{2}-\lambda^{2}}$ being a simple square root. The regular part $B_{n}^{\text {reg }}(\lambda)$ of the function $B_{N}(\lambda)$ also remains in the same limit a polynomial of order $d-3$, with the limiting form [15]:

$$
\begin{aligned}
\lim _{N \rightarrow \infty} \frac{1}{N} B_{N}(\lambda) & \equiv B \operatorname{reg}(\lambda)+\left(1-(-1)^{N}\right) \frac{\alpha}{N \lambda} \\
& =\frac{1}{2 \pi} \int_{-a}^{a} d t \frac{\lambda V^{\prime}(t)-t V^{\prime}(\lambda)}{t^{2}-\lambda^{2}} \frac{1}{\sqrt{a^{2}-t^{2}}}+\left(1-(-1)^{N}\right) \frac{\alpha}{N \lambda}
\end{aligned}
$$

Using these expressions we obtain the following result for the combination entering the differential equation eq. (3.11)

$$
\lim _{N \rightarrow \infty}\left(B_{N}(\lambda)+\frac{N}{2} V_{\alpha}^{\prime}(\lambda)\right)=N \frac{\lambda}{2 a} A(\lambda)-(-1)^{N} \frac{\alpha}{\lambda}
$$

We have kept the last term as it will give rise to two different equations for even and odd $N$. Consequently, $G_{N}(\lambda)$ can be expressed asymptotically only in terms of $A(\lambda)$ and therefore via $\rho(\lambda)$ by virtue of Eq. (4.3). We have:

$$
\begin{aligned}
& \lim _{N \rightarrow \infty} F_{N}(\lambda)=\frac{A^{\prime}(\lambda)}{A(\lambda)} \\
& \lim _{N \rightarrow \infty} G_{N}(\lambda)=N^{2} A(\lambda)^{2}\left(1-\frac{\lambda^{2}}{a^{2}}\right)+\frac{(-1)^{N} \alpha-\alpha^{2}}{\lambda^{2}}+(-1)^{N} \frac{\alpha}{\lambda} \frac{A^{\prime}(\lambda)}{A(\lambda)} .
\end{aligned}
$$

Although it seems that the first term in $G_{N}(\lambda)$ is always dominating the second term may contribute in some regimes as well, depending on the chosen rescaling of the eigenvalues which we are about to investigate in the next subsection. 


\subsection{The hard edge}

We start by defining the microscopic rescaling in the large- $N$ limit close to the hard-edge origin of the spectrum to be

$$
N \lambda \equiv \xi
$$

This amounts to magnifying the correlations at the point $\lambda \approx 0$ as $\xi$ is kept fixed while $N \rightarrow \infty$. Inserting this rescaling into the differential equations (3.12) and (3.21) and using the asymptotic expressions for the coefficients, eqs. (4.6) and (4.7) it is easy to see that the two terms with the logarithmic derivative of $A(\lambda)$ are both of subleading order $\mathcal{O}(1 / N)$. We thus obtain

$$
\varphi_{N}^{\prime \prime}(\xi)+\left(\pi^{2} \rho(0)^{2}+\frac{(-1)^{N} \alpha-\alpha^{2}}{\xi^{2}}\right) \varphi_{N}(\xi)=0, \varphi_{N} \in\left\{\psi_{N}, \chi_{N}\right\}
$$

This expression is clearly universal as the dependence on all the specific parameters $\left\{g_{2 k}\right\}$ in the polynomial potential in eq. (3.2) has been translated into a single parameter - the macroscopic spectral density at the origin $\rho(0)$. Due to the oscillating sign we have to distinguish between even and odd values of $N$, corresponding to even and odd polynomials and their Cauchy transforms. The second order differential equation has two independent solutions and it is the requirement of regularity of the solution at the origin that dictates which one to be chosen. The wave functions associated with the polynomials $\psi_{N}(\xi)$ are regular at the origin, fixing the corresponding solution to be Bessel functions of the first kind $J_{\nu}(\xi)$. On the other hand the wave functions associated with the Cauchy transforms are singular at the origin. As we will see below this makes us to choose solutions to be Bessel functions of the third kind also known as the Hankel functions, $H_{\nu}^{(1)}(\zeta)$ or $H_{\nu}^{(2)}(\zeta)$, depending on the sign of the imaginary part of the complex argument $N \epsilon \equiv \zeta$. We therefore obtain

$$
\begin{aligned}
& \psi_{2 n}(\xi) \sim(\pi \rho(0) \xi)^{\frac{1}{2}} J_{\alpha-\frac{1}{2}}(\pi \rho(0) \xi), \\
& \psi_{2 n+1}(\xi) \sim(\pi \rho(0) \xi)^{\frac{1}{2}} J_{\alpha+\frac{1}{2}}(\pi \rho(0) \xi), \\
& \chi_{2 n}(\zeta) \sim\left\{\begin{array}{rl}
(\pi \rho(0) \zeta)^{\frac{1}{2}} H_{\alpha-\frac{1}{2}}^{(1)}(\pi \rho(0) \zeta) & , \Im \mathrm{m}(\zeta)>0 \\
-(\pi \rho(0) \zeta)^{\frac{1}{2}} H_{\alpha-\frac{1}{2}}^{(2)}(\pi \rho(0) \zeta) & , \Im \mathrm{m}(\zeta)<0
\end{array},\right. \\
& \chi_{2 n+1}(\zeta) \sim\left\{\begin{array}{rl}
(\pi \rho(0) \zeta)^{\frac{1}{2}} H_{\alpha+\frac{1}{2}}^{(1)}(\pi \rho(0) \zeta) & , \Im \mathrm{m}(\zeta)>0 \\
-(\pi \rho(0) \zeta)^{\frac{1}{2}} H_{\alpha+\frac{1}{2}}^{(2)}(\pi \rho(0) \zeta) & , \Im \mathrm{m}(\zeta)<0
\end{array} .\right.
\end{aligned}
$$

In order to extract from these wave-function asymptotics the expressions for the bare polynomials and their Cauchy transforms we still have to remove the corresponding weight factor from eqs. (3.1) and (3.18) accordingly. The microscopic limit of the weight function eq. (3.2) reads:

$$
\lim _{N \rightarrow \infty} N^{2 \alpha} w(\lambda)=\xi^{2 \alpha}
$$

Multiplying or dividing the respective wavefunction by the square root of the weight function, we arrive at the final universal expressions to be inserted into the determinant of eq. (2.5):

$$
\begin{aligned}
p_{2 n}(\xi) & \sim \xi^{-\alpha+\frac{1}{2}} J_{\alpha-\frac{1}{2}}(\pi \rho(0) \xi) \\
p_{2 n+1}(\xi) & \sim \xi^{-\alpha+\frac{1}{2}} J_{\alpha+\frac{1}{2}}(\pi \rho(0) \xi)
\end{aligned}
$$




$$
\begin{aligned}
& h_{2 n}(\zeta) \sim\left\{\begin{array}{rc}
\zeta^{\alpha+\frac{1}{2}} H_{\alpha-\frac{1}{2}}^{(1)}(\pi \rho(0) \zeta) & , \Im \mathrm{m}(\zeta)>0 \\
-\zeta^{\alpha+\frac{1}{2}} H_{\alpha-\frac{1}{2}}^{(2)}(\pi \rho(0) \zeta) & , \Im \mathrm{m}(\zeta)<0
\end{array},\right. \\
& h_{2 n+1}(\zeta) \sim\left\{\begin{array}{rc}
\zeta^{\alpha+\frac{1}{2}} H_{\alpha+\frac{1}{2}}^{(1)}(\pi \rho(0) \zeta) & , \Im \mathrm{m}(\zeta)>0 \\
-\zeta^{\alpha+\frac{1}{2}} H_{\alpha+\frac{1}{2}}^{(2)}(\pi \rho(0) \zeta) & , \Im \mathrm{m}(\zeta)<0
\end{array} .\right.
\end{aligned}
$$

Let us now justify our choice of the type of Bessel functions featuring in eq. (4.11). Due to the singularity at the origin we have to choose between Bessel functions of second kind (also known as Weber functions), $Y_{\nu}(\zeta)$, and the Hankel functions. We give two independent arguments in favor of the latter choice. The first reason for selecting the Hankel functions is that it allows one to match the known "bulk" asymptotic result 25] after taking the asymptotic limit $\zeta \rightarrow \infty$. While the Weber functions would asymptotically produce sine and cosine terms (as the Bessel functions of first kind do), the Cauchy transforms in the bulk were shown to approach $\exp [ \pm i \zeta]$ [25]. This asymptotic is correctly reproduced by the Hankel functions. The second argument is related with the necessity to distinguish between the two types of the Hankel functions $H_{\nu}^{(1)}(\zeta)$ and $H_{\nu}^{(2)}(\zeta)$ according to the sign of $\Im \mathrm{m}(\zeta)$.

In fact, for $\alpha=0$ and Gaussian potential $w(\lambda)=\exp \left[-N \lambda^{2}\right]$ one can explicitly write down the integral representations for the polynomials and Cauchy transforms at finite- $N$, as discussed in 11]. Switching back to the monic normalization the polynomials are the Hermite polynomials $\pi_{n}(\lambda)=H_{n}(\lambda \sqrt{N}) /(2 \sqrt{N})^{n}$. It follows that

$$
\begin{aligned}
\pi_{2 m}(\lambda) & =\mathrm{e}^{N \lambda^{2}}(-1)^{m} 2 N \sqrt{\lambda} \int_{0}^{\infty} d s \mathrm{e}^{-N s^{2}} s^{2 m+\frac{1}{2}} J_{-\frac{1}{2}}(2 N s \lambda) \\
\pi_{2 m+1}(\lambda) & =\mathrm{e}^{N \lambda^{2}}(-1)^{m} 2 N \sqrt{\lambda} \int_{0}^{\infty} d s \mathrm{e}^{-N s^{2}} s^{2 m+\frac{3}{2}} J_{+\frac{1}{2}}(2 N s \lambda),
\end{aligned}
$$

where we have used the standard integral representation of the Hermite polynomials as well as reexpressed cosine and sine by the half-integer Bessel functions $J_{ \pm \frac{1}{2}}$. As a consistency check one can verify that eq. (4.13) at $\alpha=0$ follows from a saddle point approximation in the microscopic large- $N$ limit, remembering that $\pi \rho(0)=\sqrt{2}$ for the Gaussian potential. Using eq. (4.15) we obtain a finite- $N$ integral representation of the Cauchy transforms as follows. We can rewrite

$$
\frac{1}{x-\epsilon}=\operatorname{sgn}[\Im \mathrm{m}(\epsilon)] i \int_{0}^{\infty} d t \exp [-\operatorname{sgn}[\Im \mathrm{m}(\epsilon)] i t(x-\epsilon)], \quad \Im \mathrm{m}(\epsilon) \neq 0 .
$$

Inserting eqs. (4.15) and (4.16) into the definition (2.4) we can first perform the integral over $\lambda$ and then the one over $t$, arriving at

$$
\begin{array}{r}
\vartheta_{2 m}(\epsilon)=(-1)^{m} N \sqrt{\epsilon} \int_{0}^{\infty} d s \mathrm{e}^{-N s^{2}} s^{2 m+\frac{1}{2}}\left\{\begin{array}{rr}
H_{-\frac{1}{2}}^{(1)}(2 N s \epsilon) & , \Im \mathrm{m}(\epsilon)>0 \\
-H_{-\frac{1}{2}}^{(2)}(2 N s \epsilon) & , \Im \mathrm{m}(\epsilon)<0
\end{array},\right. \\
\vartheta_{2 m+1}(\epsilon)=(-1)^{m} N \sqrt{\epsilon} \int_{0}^{\infty} d s \mathrm{e}^{-N s^{2}} s^{2 m+\frac{3}{2}}\left\{\begin{array}{rr}
H_{+\frac{1}{2}}^{(1)}(2 N s \epsilon) & , \Im \mathrm{m}(\epsilon)>0 \\
-H_{+\frac{1}{2}}^{(2)}(2 N s \epsilon) & , \Im \mathrm{m}(\epsilon)<0
\end{array} .\right.
\end{array}
$$

In taking the microscopic large- $N$ limit we again recover eq. (4.14) at $\alpha=0$ from a saddle point approximation. This fact confirms the correct choice of the solution for the Cauchy transform and thus for the wavefunctions eq. (4.11) for a Gaussian potential with $\alpha=0$. Since the parameters $\alpha$ as well as the higher order coupling constants $g_{2 k}$ in the potential eq. (3.3) are real and can be switched 
on smoothly the choice of the type of Hankel function remains fixed for a general potential for general $\alpha$ as well.

Now we can use the relations between the polynomials and Cauchy transforms of the chiral and nonchiral ensemble, eqs. (3.25) and (3.31) respectively, to read off immediately the universal asymptotic formulas for the chiral Unitary Ensemble from (4.13) and (4.14):

$$
\begin{aligned}
& p_{n, c h}^{(\alpha)}\left(\xi^{2}\right) \sim \xi^{-\alpha} J_{\alpha}(\pi \rho(0) \xi), \\
& h_{n, c h}^{(\alpha)}\left(\zeta^{2}\right) \sim\left\{\begin{array}{rl}
\zeta^{\alpha} H_{\alpha}^{(1)}(\pi \rho(0) \zeta) & , \quad \Im \mathrm{m}(\zeta)>0 \\
-\zeta^{\alpha} H_{\alpha}^{(2)}(\pi \rho(0) \zeta) & , \quad \Im \mathrm{m}(\zeta)<0
\end{array} .\right.
\end{aligned}
$$

We see that for integer values of $\alpha$ the indices of the Bessel functions are also integer, compared to half integer values for the non-chiral case above. To fix independently the correct Bessel solution corresponding to the sign of $\Im \mathrm{m}(\xi)$ we can repeat the argument as in eqs. (4.15) and (4.17), but this time for general $\alpha \neq 0$. The corresponding finite- $N$ expression have already been given in [11, following the integral representation of generalized Laguerre polynomials, and we only repeat them here:

$$
\begin{aligned}
& \pi_{n, c h}\left(x^{2}\right)=\mathrm{e}^{N x^{2}}(-1)^{n} N x^{-\alpha} \int_{0}^{\infty} d t \mathrm{e}^{-N t} t^{n+\alpha / 2} J_{\alpha}(2 N x \sqrt{t}), \\
& \vartheta_{n, c h}\left(\epsilon^{2}\right)=\frac{(-1)^{n}}{2} N \epsilon^{\alpha} \int_{0}^{\infty} d t \mathrm{e}^{-N t} t^{n+\alpha / 2}\left\{\begin{array}{rr}
H_{\alpha}^{(1)}(2 N \epsilon \sqrt{t}) & , \quad \mathrm{m}(\epsilon)>0 \\
-H_{\alpha}^{(2)}(2 N \epsilon \sqrt{t}) & , \Im \mathrm{m}(\epsilon)<0
\end{array} .\right.
\end{aligned}
$$

The saddle point approximation reconfirms eq. (4.19) for the Gaussian potential $V_{\alpha}^{c h}(\lambda)=\lambda-\frac{\alpha}{N} \ln (\lambda)$ and, by invoking the continuity argument for a general potential as well.

\subsection{The bulk of the spectrum}

In this section we briefly describe the microscopic limit in the bulk of the spectrum, which amounts to setting $\alpha=0$ in the differential equation (4.9). For the non-chiral case the corresponding formulae match with those obtained in [25].

It is evident that when logarithmic terms are absent the origin of the spectrum is no longer singled out. Under these conditions correlation functions when properly scaled should be the same at every point inside the support of the spectrum, due to translational invariance. More precisely, let us pick a fixed value $\lambda_{*} \in(-a, a)$, that means neither at the origin nor at the edge of the support. The microscopic rescaling around the chosen point is then defined as

$$
N\left(\lambda-\lambda_{*}\right) \equiv \xi
$$

Inserting this expression into that for the asymptotic coefficients, eqs. (4.6) and (4.7), we can easily see that only the first term in $G_{N}(\lambda)$ contributes yielding

$$
\varphi_{N}^{\prime \prime}(\xi)+\pi^{2} \rho\left(\lambda_{*}\right)^{2} \varphi_{N}(\xi)=0, \varphi_{N} \in\left\{\psi_{N}, \chi_{N}\right\}
$$

where we used eq. (4.3) squared. This is just eq. (4.9) with $\alpha=0$, replacing $\rho(0)$ by the universal parameter $\rho\left(\lambda_{*}\right)$. From eqs. (4.10) and (4.11) we see that the solutions are Bessel functions of index $\pm \frac{1}{2}$, which are just sine and cosine. The differential equation no longer distinguishes between even and odd $N$, but the parity fixes the solution uniquely. The solutions for the asymptotic Cauchy 
transform will no longer be singular as we expand around the point $\lambda=\lambda_{*}$. However, we can still use the results of the previous subsection for $\alpha=0$ in order to fix the solution uniquely depending on the sign $\Im \mathrm{m}(\epsilon)$ in the Cauchy transform. Omitting the constant proportionality factor $w\left(\lambda_{*}\right)^{ \pm \frac{1}{2}}$ when switching from wavefunctions to polynomials and their Cauchy transforms yields the explicit expressions for the latter:

$$
\begin{aligned}
& p_{2 n}(\xi) \sim \cos \left(\pi \rho\left(\lambda_{*}\right) \xi\right), \\
& p_{2 n+1}(\xi) \sim \sin \left(\pi \rho\left(\lambda_{*}\right) \xi\right) \\
& h_{2 n}(\zeta) \sim\left\{\begin{array}{rl}
\exp \left(+i \pi \rho\left(\lambda_{*}\right) \zeta\right) & , \quad \Im \mathrm{m}(\zeta)>0 \\
-\exp \left(-i \pi \rho\left(\lambda_{*}\right) \zeta\right) & , \quad \Im \mathrm{m}(\zeta)<0
\end{array},\right. \\
& h_{2 n+1}(\zeta) \sim\left\{\begin{array}{ll}
-i \exp \left(+i \pi \rho\left(\lambda_{*}\right) \zeta\right) & , \quad \Im \mathrm{m}(\zeta)>0 \\
-i \exp \left(-i \pi \rho\left(\lambda_{*}\right) \zeta\right) & , \quad \Im \mathrm{m}(\zeta)<0
\end{array} .\right.
\end{aligned}
$$

Due to the fact that no $\alpha$-dependence is present in eq. (4.22) the polynomials and Cauchy transform in the chiral ensemble are identical to the even half of the non-chiral ones. The eqs. (3.25) and (3.31) then give:

$$
\begin{aligned}
p_{n, c h}^{(\alpha)}\left(\xi^{2}\right) & \sim \cos \left(\pi \rho\left(\lambda_{*}\right) \xi\right) \\
h_{n, c h}^{(\alpha)}\left(\zeta^{2}\right) & \sim\left\{\begin{array}{rr}
\zeta^{-1} \exp \left(+i \pi \rho\left(\lambda_{*}\right) \zeta\right) & , \quad \Im \mathrm{m}(\zeta)>0 \\
-\zeta^{-1} \exp \left(-i \pi \rho\left(\lambda_{*}\right) \zeta\right) & , \quad \Im \mathrm{m}(\zeta)<0
\end{array} .\right.
\end{aligned}
$$

\subsection{The soft edge}

Finally we turn our attention to the correlation functions in the vicinity of the edges $\lambda= \pm a$ of the support of the spectrum, the so-called soft edges [32, 33]. Restricting ourselves to $\lambda=+a$ for symmetry reasons we define the microscopic scaling limit at the soft edge as

$$
N^{\frac{2}{3}}(\lambda-a) \equiv \xi
$$

In this way we magnify the region around the endpoint of the support. For negative (positive) $\xi$ we are inside (outside) the support. In the limit eq. (4.27) only the first term in eq. (4.7) contributes and the different power in $N$ is due to the factor $\left(a^{2}-\lambda^{2}\right)$ that multiplies $A(\lambda)^{2}$. In the large- $N$ limit the differential equation acquires the form:

$$
\varphi_{N}^{\prime \prime}(\xi)-\xi \frac{2 A(a)^{2}}{a} \varphi_{N}(\xi)=0, \varphi_{N} \in\left\{\psi_{N}, \chi_{N}\right\}
$$

It is again universal, extending the results of [17 to the Cauchy transforms. After changing variables

the solutions is obtained in terms of the Airy functions. Again omitting the prefactors $w(a)^{ \pm \frac{1}{2}}$ when changing from wavefunctions to the original polynomials and Cauchy transforms we obtain:

$$
\begin{aligned}
& p_{N}(\xi) \sim \operatorname{Ai}\left(\left(2 a^{-1} A(a)^{2}\right)^{\frac{1}{3}} \xi\right) \\
& h_{N}(\zeta) \sim\left\{\begin{array}{rr}
-\mathrm{e}^{+\frac{2 \pi i}{3}} \operatorname{Ai}\left(\left(2 a^{-1} A(a)^{2}\right)^{\frac{1}{3}} \zeta \mathrm{e}^{+\frac{2 \pi i}{3}}\right), & \Im \mathrm{m}(\zeta)>0 \\
\mathrm{e}^{-\frac{2 \pi i}{3}} \operatorname{Ai}\left(\left(2 a^{-1} A(a)^{2}\right)^{\frac{1}{3}} \zeta \mathrm{e}^{-\frac{2 \pi i}{3}}\right) & , \Im \mathrm{m}(\zeta)<0
\end{array} .\right.
\end{aligned}
$$


Here we have chosen for the Cauchy transforms one of the two solutions of the Airy equation, that one which is linearly independent of $\operatorname{Ai}(x)$ [35], in coordination with the sign of $\Im \mathrm{m}(\zeta)$ :

$$
\operatorname{Ai}\left(z \mathrm{e}^{ \pm \frac{2 \pi i}{3}}\right)=\frac{1}{2} \mathrm{e}^{ \pm \frac{\pi i}{3}}(\operatorname{Ai}(z) \mp i \operatorname{Bi}(z))
$$

The functions $\operatorname{Bi}(z)$ is a new feature emerging when negative moments of the characteristic polynomials are considered. For positive moments only [33], as well as for the spectral densities and related objects [12, 32, 34] only functions $\operatorname{Ai}(z)$ feature in the final results.

The discontinuity between the two solutions is again a simple Airy function, due to the functional relation [35]:

$$
\operatorname{Ai}(z)=-\mathrm{e}^{+\frac{2 \pi i}{3}} \operatorname{Ai}\left(z \mathrm{e}^{+\frac{2 \pi i}{3}}\right)-\mathrm{e}^{-\frac{2 \pi i}{3}} \operatorname{Ai}\left(z \mathrm{e}^{-\frac{2 \pi i}{3}}\right)
$$

For real negative argument the Airy functions $\mathrm{Ai}$ and $\mathrm{Bi}$ are oscillating while for positive argument they monotonously decrease/increase, respectively. For large positive argument we conclude that

$$
\lim _{z \rightarrow+\infty} \mp \mathrm{e}^{ \pm \frac{2 \pi i}{3}} \operatorname{Ai}\left(z \mathrm{e}^{ \pm \frac{2 \pi i}{3}}\right)=-i \operatorname{Bi}(z)
$$

and thus see that both solutions eq. (4.30) agree. This fixes our choice of the boundary condition as for large positive arguments outside the support the Cauchy transforms should no longer experience a discontinuity across the real line.

The solution for even and odd $N$ is eqs. (4.29) and (4.30) are the same. This immediately implies that the results for the UE and the chUE are identical, as must be the case far from the origin of the spectrum. Therefore we have to compute also the difference between $\varphi_{N}$ and $\varphi_{N-1}$ as they both appear in expressions relating the correlation functions containing ratios of characteristic polynomials to orthogonal polynomials and their Cauchy transform ${ }^{3}$. For that aim we use explicitly the differentiation eqs. (3.8) and (3.20) for the corresponding wave functions together with the limiting relation eq. (4.4). Following [30] we finally obtain:

$$
\varphi_{N-1}(\xi)=\varphi_{N}(\xi)+\frac{1}{N^{\frac{1}{3}} A(a)} \varphi_{N}^{\prime}(\xi), \quad \varphi_{N} \in\left\{\psi_{N}, \chi_{N}\right\}
$$

This completes our demonstration of universality for the orthogonal polynomials and their Cauchy transforms for both the UE and chUE, in all three different regions of the spectrum.

We close with the following comment. Once the universality of expectation values of ratios of characteristic polynomials eq. (2.2) is demonstrated it immediately translates to that of all other correlation functions that are generated by them by differentiations. As a check we can consider the correlation function of the eigenvalue densities by using eqs. (4.13) - (4.14) eqs. (4.18) - (4.19) respectively, and taking necessary derivatives and discontinuities. In this way one reproduces the known universal microscopic spectral densities of the unitary and chiral unitary ensemble, respectively, see e.g. 14. It covers the bulk limit by setting $\alpha=0$. The difference is that in the chUE the resolvent has a true singularity at the origin while in the UE the discontinuity originates from patching two different solutions, each of which is regular at this point. This is another way of seeing that for the UE the "old" replica limit using saddle point techniques is only able to reproduce the asymptotic expansions [36] while for the chiral case it yields the exact result [37. Similar checks for the universal microscopic eigenvalue density at the soft edge can be performed using eqs. (4.29) and (4.30), recovering known expression from [17].

\footnotetext{
${ }^{3}$ The same remark in fact applies already to the chUE in the previous subsections.
} 


\section{Discussion}

For a broad class of measures we have shown that the Cauchy transforms of orthogonal polynomials display properties very similar to those of the polynomials themselves. In particular, they obey the same three step recursion relation. The wave functions associated with the Cauchy transforms satisfy the same second order differential equation as those associated with the polynomials. This correspondence holds for finite- $N$ with polynomial potentials of arbitrary but fixed even degree $d$, provided that $N>d-2$. In the microscopic large- $N$ limit we have thus obtained the same universal Bessel equation at the hard edge, and the Airy equation at the soft edge of the spectrum. For the Cauchy transform the choice of the correct solutions is dictated by their singularity at the origin and the sign of the imaginary part of the argument. This identification was also supported by a comparison with exact integral representations available for the Gaussian weight at finite- $N$.

Using the general results [10 the universality of arbitrary ratios of characteristic polynomials in RMT with unitary invariance thus follows. Our consideration complements the rigorous proof 25] valid for the bulk of the spectrum and relied on the Riemann-Hilbert approach. The arguments presented here rely on the previous results in 30, 21. Although partly heuristic by strict mathematical standards, the method has the clear advantage of being much more transparent and able to treat both cases of the chUE and the UE on equal footing.

Since our differential equation for the wavefunctions is valid at finite- $N$ we could be tempted to take the macroscopic large- $N$ limit as well. It gives the eigenvalue correlations at distances large compared to the mean level spacing, where the local fluctuations are smoothed. While the spectral density itself is non-universal, all higher order connected correlation functions are known to be universal [38. It has been shown in 30, that the density and the connected two point function can be also obtained from smoothing products of orthogonal polynomials. However, for higher order correlations the corrections to the orthogonal polynomials become extremely difficult to evaluate and are unknown so far. For that reason we have not tried to consider smoothened Cauchy transforms in order to recover the known one and two-point function. It is also less clear if the procedures of smoothening and taking the discontinuity after differentiation can be interchanged safely.

Finally it would be very interesting to extend the general results [10] to matrix models with complex eigenvalues and to prove their universality. First results in this direction have already been obtained 39]. There, arbitrary products of characteristic polynomials have been expressed in a determinant formula, which allowed to demonstrate their universality at the regime of weak non-Hermiticity.

Acknowledgments: We wish to thank Graziano Vernizzi and Boris Khoruzhenko for a critical reading and comments on the manuscript. This work was supported by Brunel University Vice Chancellor Grant (YVF) and by the Heisenberg fellowship of the Deutsche Forschungsgemeinschaft (GA).

\section{References}

[1] E.V. Shuryak and J.J.M. Verbaarschot, Nucl. Phys. A560 (1993) 306 hep-th/9212088; J. Verbaarschot, Phys. Rev. Lett. 72 (1994) 2531 hep-th/9401059.

[2] A.V. Andreev and B.D. Simons, Phys. Rev. Lett. 75 (1995) 2304.

[3] J.P. Keating and N. Snaith, Commun. Math. Phys. 214 (2000) 57.

[4] E. Brézin and S. Hikami, Commun. Math. Phys. 214 (2000) 111 math-ph/9910005. 
[5] P.H. Damgaard, Phys. Lett. B424 (1998) 322 hep-th/9711110; G. Akemann and P.H. Damgaard, Nucl. Phys. B528 (1998) 411 hep-th/9801133; Phys. Lett. B432 (1998) 390 hep-th/9802174.

[6] J.C. Osborn, D. Toublan and J.J.M. Verbaarschot, Nucl. Phys. B540 (1999) 317 hep-th/9806110; P.H. Damgaard, J.C. Osborn, D. Toublan and J.J.M. Verbaarschot, Nucl. Phys. B547 (1999) 305 hep-th/9811212.

[7] K. Splittorff and J.J.M. Verbaarschot Phys. Rev. Lett. 90, 041601 (2003) cond-mat/0209594.

[8] E. Kanzieper, Phys. Rev. Lett. 89, 250201 (2002) cond-mat/0207745.

[9] Y.V. Fyodorov and E. Strahov, Nucl. Phys. B647 (2002) 581 hep-th/0205215.

[10] Y.V. Fyodorov and E. Strahov, math-ph/0204051

[11] Y.V. Fyodorov and G. Akemann, (to appear in JETP Letters) cond-mat/0210647.

[12] M.L. Mehta, Random Matrices, Second Edition, Academic Press, London 1991.

[13] E. Brézin and A. Zee, Nucl. Phys. B402 (1993) 613.

[14] G. Akemann, P.H. Damgaard, U. Magnea and S. Nishigaki, Nucl. Phys. B487 (1997) 721 hep-th/9609174.

[15] E. Kanzieper and V. Freilikher, Phil. Mag. B77 (1998) 1161 cond-mat/9704149.

[16] P.H. Damgaard and S.M. Nishigaki, Nucl. Phys. B518 (1998) 495 hep-th/9711023; Phys. Rev. D57 (1998) 5299 hep-th/9711096.

[17] E. Kanzieper and V. Freilikher, Phys. Rev. Lett. 78 (1997) 3806 chao-dyn/9701006.

[18] G. Akemann, P.H. Damgaard, U. Magnea and S.M. Nishigaki, Nucl. Phys. B519 (1998) 628 hep-th/9712006.

[19] G. Akemann and G. Vernizzi, Nucl. Phys. B631 (2002) 471 hep-th/0201165.

[20] L. Pastur and M. Shcherbina, J. Stat. Phys. 86 (1997) 109.

[21] P. Deift, T. Kriecherbauer, K.T.-R. McLaughlin, S. Venakides and X. Zhou, Comm. Pure and Appl. Math. Vol. 52 (1999) 1335; ibid. 52 (1999) 1491.

[22] P. Bleher and A. Its, Ann. of Math. 150 (1999) 185 math-ph/9907025.

[23] P. Deift, Orthogonal Polynomials and Random Matrices (Courant Lecture Notes v.3), Providence R.I., American Mathematical Society 2000.

[24] P.H. Damgaard, Proceedings of "Quantum Chromodynamics 98", Paris 1998, p. 296-303 hep-th/9807026.

[25] E. Strahov and Y.V. Fyodorov, math-ph/0210010.

[26] K.B. Efetov, Supersymmetry in Disorder and Chaos, Cambridge University Press, Cambridge 1997.

[27] T. Guhr, J. Math. Phys. 32 (1991) 336. 
[28] Y.V. Fyodorov, Nucl. Phys. B621 [PM] (2002) 643 math-ph/0106006.

[29] P.J. Forrester, Nucl. Phys. B402 (1993) 709; T. Nagao and P.J. Forrester, Nucl. Phys. B435 (1995) 401.

[30] E. Kanzieper and V. Freilikher, NATO ASI, Series C (Math. and Phys. Sciences), Vol. 531, p. 165 - 211, Kluwer, Dordrecht 1999 cond-mat/9809365.

[31] P. Bleher and B. Eynard, hep-th/0209087.

[32] C.A. Tracy and H. Widom, Commun. Math. Phys. 159 (1994) 151; ibid 161 (1994) 289; ibid 163 (1994) 33.

[33] E. Brézin and S. Hikami, Phys. Rev. E62 (2000) 3558 math-ph/0004018.

[34] P.J. Forrester and N.S. Witte, Commun. Math. Phys. 219 (2001) 357 math-ph/0103025.

[35] M. Abramowitz and I.A. Stegun, Handbook of Mathematical Functions, Dover Publ., New York 1972.

[36] D. Dalmazi and J.J.M Verbaarschot Nucl. Phys. B592 (2001) 419 hep-th/0005229.

[37] G. Akemann, D. Dalmazi, P.H. Damgaard and J.J.M. Verbaarschot, Nucl. Phys. B601 (2001) 77 hep-th/0011072.

[38] J. Ambjørn, J. Jurkiewicz and Y. Makeenko, Phys. Lett. 251 (1990) 517; J. Ambjørn, L. Chekhov, C. F. Kristjansen and Yu. Makeenko, Nucl. Phys. 404 (1993) 127 hep-th/9302014.

[39] G. Akemann and G. Vernizzi, Nucl. Phys. B in press hep-th/0212051. 